\title{
Direct Visualisation of Abnormal Dendritic Spine Morphology in the Hippocampus of the R6/2 Transgenic Mouse Model of Huntington's Disease
}

\author{
Simon J. Bulley, Cheney J.G. Drew and A. Jennifer Morton* \\ Department of Pharmacology, University of Cambridge, Tennis Court Road, Cambridge, UK
}

\begin{abstract}
.
Background: Spatial learning deficits are observed in R6/2 mice, a transgenic mouse model of Huntington's Disease (HD). Spatial learning is a hippocampal-dependent process, and impairment of memory is thought to be due, at least in part, to structural changes such as loss of dendritic spines.

Objective: To analyse dendritic spines in the hippocampus of R6/2 mice to determine if there are changes that correlate with the hippocampal dysfunction observed in these mice.

Methods: A double transgenic cross between R6/2 mice and a reporter line (YFP-H) of mice that express yellow fluorescent protein (YFP) in a subset of their neurons was used. This allowed us to visualise dendritic spines in the brains of R6/2 mice directly.

Results: Clear differences were seen in the distribution of YFP in the hippocampal formation of wild-type (WT)-YFP-H and R6/2-YFP-H mice, particularly in the CA1 region. We quantified dendritic spine density and dendritic spine length in the apical dendrites of the CA1 hippocampal neurons. A significant reduction in dendritic spine density, and a concomitant increase in dendritic spine length was observed in R6/2-YFP-H mice compared to WT-YFP-H mice.

Conclusion: The R6/2-YFP-H mouse is a useful tool for directly visualising dendritic spines in the brain of a Huntington's disease mouse model. The changes we observed in dendritic spine density and length in the hippocampus might contribute to the synaptic plasticity deficits and behavioural alteration of impaired spatial learning seen in R6/2 mice.
\end{abstract}

Keywords: Dendritic spines, learning, long-term potentiation, LTP, confocal microscopy, green fluorescent proteins, fragile X syndrome

\section{INTRODUCTION}

Huntington's disease (HD) is an autosomal dominant progressive neurodegenerative disorder that is characterised by motor, cognitive, and emotional

${ }^{*}$ Correspondence to: A. Jennifer Morton, Department of Pharmacology, University of Cambridge, Tennis Court Road, Cambridge CB2 1PD, UK. Tel.: +44 1223 334000; E-mail: ajm41@ cam.ac.uk. deficits [1]. It is caused by an expanded CAG repeat in the HTT gene. The R6/2 mouse model of HD expresses the first exon of $H T T$ with an expanded $\mathrm{CAG}$ repeat [2]. Spatial learning deficits are observed in R6/2 mice in the Morris water maze [3]. Spatial learning is a hippocampal-dependent process $[4,5]$ that involves a form of synaptic plasticity known as long-term potentiation (LTP) [6]. Impaired synaptic plasticity is observed in the CA1 region of the R6/2 
mouse hippocampus [7]. It has been shown in cultured hippocampal neurons that dendritic spines in proximity to huntingtin (htt) protein aggregates are lost because of a functional defect in local endosomal recycling [8], and that striatal excitatory synapses are significantly reduced in YAC128 mice [9]. Changes in spine density or spine length are thought to represent a morphological correlate of altered brain functions associated with learning and memory [10]. LTP is associated with increased spine densities [11] and with the formation of new synapses [12]. Interestingly, the pronounced neurological phenotype that is observed in the R6/2 mouse occurs largely in the absence of neurodegeneration [2], suggesting that neuronal loss may be secondary to neuronal dysfunction. This may also be the case in the early stages of the human disease, since both motor $[13,14]$ and cognitive deficits $[15,16]$ can be detected in patients before neurodegenerative changes are seen. It therefore seems possible that some of the cognitive symptoms in HD are caused by changes in dendritic spine density and length in the hippocampus prior to neuronal cell death.

Here we crossed R6/2 mice with a reporter line (YFP-H) that expresses YFP in a subset of neurons [17]. This provided a Golgi-like vital stain, allowing a detailed analysis of neuronal morphology at the spine level [17]. We investigated dendritic spine density, dendritic spine morphology, and dendritic spine length in the apical CA1 dendrites of R6/2-YFP-H mice.

\section{MATERIALS AND METHODS}

\section{Animals}

All experiments were carried out in accordance with the UK Animals (Scientific Procedures) Act 1986.

R6/2 mice used in this study were obtained from colonies maintained in the Department of Pharmacology, University of Cambridge (Cambridge, UK). YFP$\mathrm{H}$ mice [17] were originally obtained from Jackson Laboratories, USA. Mice had a mixed genetic background [C57BL6J $\times(\mathrm{CBA} \times \mathrm{C} 57 \mathrm{BL6}$ F1)]. Maintenance was by backcrossing onto CBA $\times$ C57BL6 F1 female mice. All mice were housed in hard-bottomed polypropylene cages on a 12 hour light/dark cycle, maintained at $21-23^{\circ} \mathrm{C}, 55 \pm 10 \%$ humidity, and were provided with $8 / 10$ grade corncob bedding, and shredded paper nesting material. All animals were given ad libitum access to water and standard dry laboratory food. Tail biopsy was performed at 3 weeks for the purpose of genotyping [18]. Mating of male R6/2 mice with YFP-positive female mice created R6/2-YFP-H double transgenic mice. Four R6/2-YFP-H mice and three WT littermates were used. Animals were killed at 20 weeks of age, at which stage they were symptomatic, and approximately 4 weeks from end-stage. The R6/2-YFP-H mice had a CAG repeat length of $157 \pm 3$ (mean $\pm \mathrm{SEM}$ ).

\section{Tissue preparation}

All animals were perfused transcardially with $4 \%$ paraformaldehyde in $100 \mathrm{mM}$ phosphate buffered saline. Brains were cryo-protected in $30 \%$ sucrose solution, and $60 \mu \mathrm{m}$ coronal sections were cut. These sections were counter-stained with Hoechst 33258 (Sigma), and mounted in ProLong Gold (Molecular Probes, UK).

\section{Imaging}

All brightfield and epi-fluorescence images were captured using a Nikon Eclipse TE2000-S inverted microscope with a Nikon DXM 1200 digital camera (Nikon Instruments, Yokohama, Japan). Confocal images were captured using a Leica DM IRE2 inverted microscope (Leica Microsystems, Heidelberg, Germany) with an $80 \mu \mathrm{m}$ working distance $63 \times / 1$.4-numerical aperture oil-immersion objective. The LASER was set to an excitation wavelength of $514 \mathrm{~nm}$. Images were typically collected at a resolution of $1024 \times 1024$ pixels and averaged over two lines. Where stacks of $z$ sections were collected for gross hippocampal morphology, the final magnification was $63 \times$ and the scanning step size was $1.14 \mu \mathrm{m}$. Where stacks of $z$ sections were collected for quantification of dendritic spine density and dendritic spine length, the final magnification was $504 \times$ and the scanning step size $0.12 \mu \mathrm{m}$. Maximum intensity projections (MIP) of stacks of $z$ sections were produced using the software ImageJ [19].

\section{Dendritic spine analysis}

Spines on the apical dendrites of CA1 pyramidal neurons were examined. For quantification of dendritic spine density and dendritic spine length, $10 \mu \mathrm{m}$ long segments of apical CA1 dendrites were randomly chosen for analysis. Dendritic spines projecting to the sides of dendrites were counted, and their morphology classified, from MIP images. Dendritic spines lying above and below dendrites could not be analysed from MIP images as they were obscured by the fluorescence from 
the dendrite. They were therefore counted and classified from the individual images within the stacks in which they were visible. Spines were subclassified into one of five morphologies (mushroom-shaped, stubby, cup-shaped, thin, and filopodia) according to the classification described in [20]. Mushroom, thin, and stubby morphologies were assigned based on the ratio of spine head diameter $(h)$ to spine neck diameter $(n)$ according to the following criteria: mushroom: $h>2 n$, thin: $h<2 n$, stubby: $h<n$ [21]. All spines were counted on 135 dendrite segments from three WT-YFP-H mice (45 dendrite segments per animal; 3111 spines in total), and 180 dendrite segments from four R6/2-YFP-H mice (45 dendrite segments per animal; 2688 spines in total). Dendritic spine analysis was conducted blind to mouse genotype from randomly selected coded MIP images. Data are expressed as mean \pm standard error of the mean. Spine lengths were measured using the software ImageJ [19]. Spine lengths were measured from the shaft of the dendrite to the tip of the spine head. Only the length of mushroom shaped spines was measured.

\section{Statistical analysis}

The data were assessed by two-tailed unpaired $t$ tests. $\mathrm{N}$ was the number of animals in each group. Statistical analysis was performed using GraphPad Prism version 3.02 for Windows (GraphPad Software, San Diego, CA). A critical value for significance of $P<0.05$ was used throughout the study.

\section{RESULTS}

Gross morphological analysis of brains from WT-YFP-H and R6/2-YFP-H mice

The global pattern of YFP expression was similar in WT-YFP-H mice and R6/2-YFP-H mice (Fig. 1), although as expected [2, 22] the brains of R6/2-YFP-H mice were smaller than those of WT-YFP-H mice. We originally planned to look at spines in the striatum, but YFP was not expressed in a number of brain regions, including the striatum. The striatum would otherwise have been an area of great interest as spine changes in striatal dendrites have previously been reported [23]. In the brain regions in which YFP was expressed, there was no consistent difference in the level of expression between WT-YFP-H and R6/2-YFP-H mice except in the hippocampus (Fig. 1). In the hippocampus of R6/2-YFP-H mice, levels of YFP were qualitatively lower in the CA1 hippocampal field, and higher in the dentate gyrus when compared with WT-YFP-H mice (Fig. 1A, B). High magnification confocal microscopy images of the hippocampal formation of R6/2-YFP-H and WT-YFP-H mice confirmed this finding (Fig. 1C, D). Anti-ubiquitin immunocytochemistry revealed that inclusions were present in the CA1 neurons of R6/2YFP-H mice, but not in the CA1 neurons of WT-YFP-H mice (data not shown).

\section{R6/2-YFP-H mice have reduced hippocampal spine density and increased hippocampal spine length}

We wondered if the changes in the CA1 hippocampal field were due to changes in spine density. Therefore we quantified dendritic spine density and dendritic spine length in the apical CA1 dendrites of WT-YFP-H and R6/2-YFP-H mice. Examples of $10 \mu \mathrm{m}$ apical CA1 dendrite segments from WT-YFP-H and R6/2-YFP-H mice shown in Figs. 2A-B illustrate the appearance of spines, the variability of the spine density, and the range of dendrite diameters examined. Using the confocal microscope we followed neurons from soma to dendrite, and found no evidence of areas of defective filling of dendrites with YFP. The morphological classification of dendritic spines employed is shown in Fig. 2C. There was a significant decrease in spine density in R6/2-YFP-H mice when only mushroomshaped spines were considered $(P<0.01$, Fig. 3$)$ and when all spines were considered $(P<0.05$, Fig. 3$)$. There was no significant difference in spine density between R6/2-YFP-H and WT-YFP-H mice when only filopodia, thin, stubby, or cup-shaped spines were considered (Fig. 3). This indicates that the overall decrease in spine density in R6/2-YFP-H mice was mainly due to a decrease in the density of mushroomshaped spines. There was a significant increase in mean spine length in R6/2-YFP-H mouse brains $(0.83 \mu \mathrm{m} \pm 0.011 \mu \mathrm{m}, N=966)$ compared to those seen in WT-YFP-H brains $(0.67 \mu \mathrm{m} \pm 0.0085 \mu \mathrm{m}$, $N=1276, P<0.01$ ) (see Fig. $1 \mathrm{G}, \mathrm{H}$ ). The mean diameters of the dendrite segments used for quantifying spines were not significantly different between the WT-YFP-H $(1.1 \mu \mathrm{m} \pm 0.066 \mu \mathrm{m}, N=135)$ and R6/2-YFP-H $(1.0 \mu \mathrm{m} \pm 0.052 \mu \mathrm{m}, N=180, P=0.22)$ groups. Although we classified spines according to the criteria already set out [20], certain morphologies have been proposed to be characteristic of immature' spines, and certain others to be characteristic of 'mature' spines [24]. The increased spine length we observed in R6/2-YFP-H mice is characteristic of 


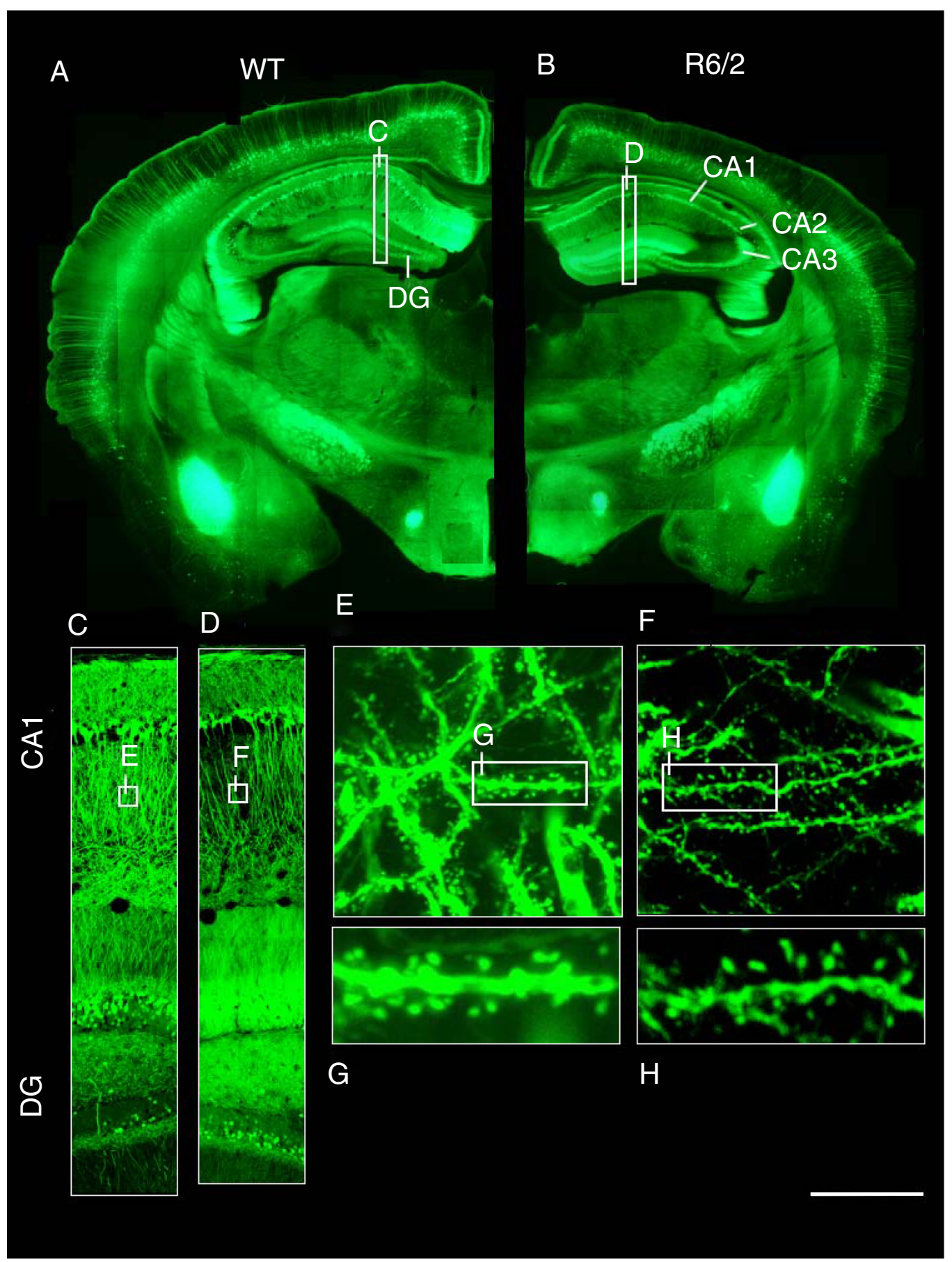

Fig. 1. The pattern of expression of YFP. Epi-fluorescence (A, B) and confocal (C-H) images from WT-YFP-H (A, C, E, G) and R6/2-YFP-H $(\mathrm{B}, \mathrm{D}, \mathrm{F}, \mathrm{H})$ brain sections taken at the level of the hippocampus showing the distribution of YFP in neurons. Scale bar represents $1 \mathrm{~mm}$ for panels $A$ and $B, 200 \mu \mathrm{m}$ for panels $C$ and $D, 10 \mu \mathrm{m}$ for panels $E$ and $F$, and $4 \mu \mathrm{m}$ for panels $\mathrm{G}$ and $\mathrm{H}$.

immature spines [24]. Filopodia, in particular long filopodia, are also thought to represent immature spines [24]. We did not have sufficient data for a meaningful statistical analysis of filopodia between R6/2-YFP$\mathrm{H}$ and WT-YFP-H mice. A qualitative impression of the spines encountered in the present study, how- 
A

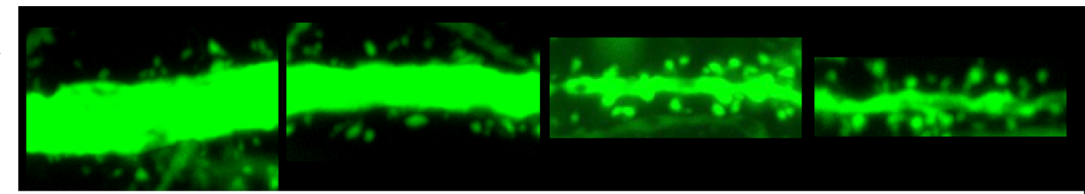

B

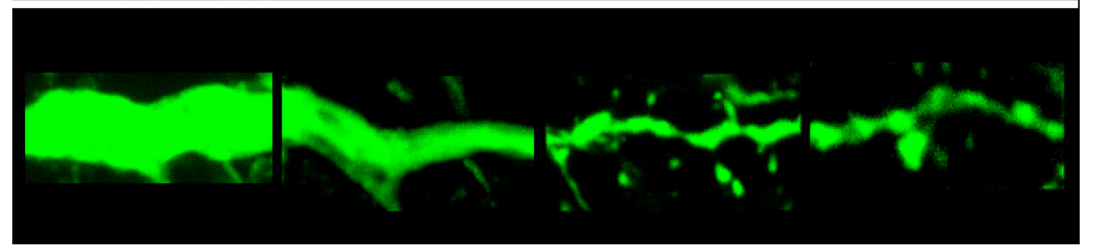

C

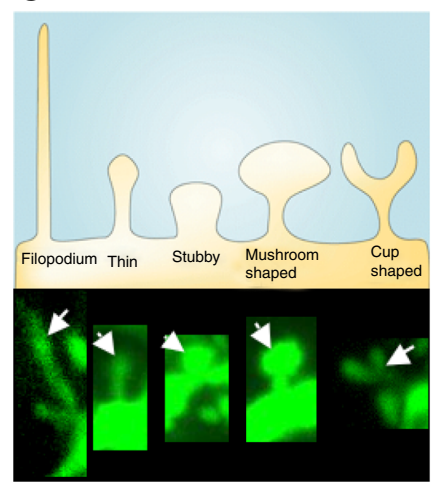

$\mathrm{D}$

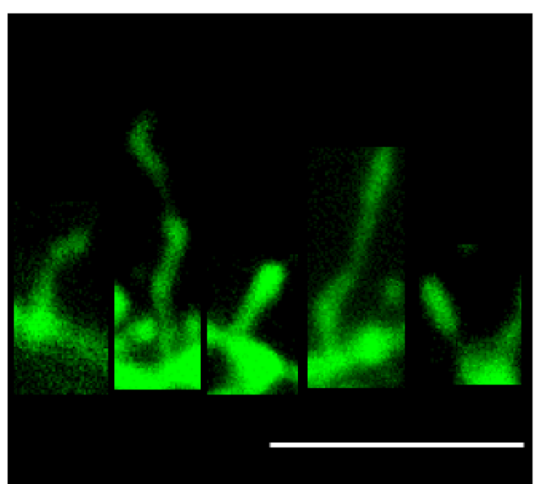

Fig. 2. Classification of spines. Examples of $10 \mu \mathrm{m}$ apical CA1 dendrite segments; typical images from (A) a WT-YFP-H mouse, and (B) a R6/2-YFP-H mouse. Each row of images is composed of four separate maximum intensity projection images of $10 \mu \mathrm{m}$ dendrite segments. The images illustrate the appearance of spines, the variability of spine density, and the range of dendrite diameters examined. The morphological classification of dendritic spines is shown in panel C. (Cartoon reprinted by permission from Macmillan Publishers Ltd: Nature Reviews Neuroscience, 2, 880 - 888, copyright 2001). Examples of normal spines are shown in C; examples of spines with immature morphologies from R6/2-YFP-H mice are shown in D. The entire z-stack of available images was examined to ensure that the structures presented in D are spines rather than dendrite branches. The scale bar in panel 2D represents $10 \mu \mathrm{m}$ in panels $\mathrm{A}$ and $\mathrm{B}$, and $5 \mu \mathrm{m}$ in panel $\mathrm{D}$.

ever, is that immature morphologies such as very long filopodia are seen more often in R6/2-YFP-H mice than in WT-YFP-H mice (Fig. 2D).

\section{DISCUSSION}

Here we used an R6/2-YFP-H double transgenic mouse model of HD to quantify dendritic spine density and dendritic spine length in the apical dendrites of the CA1 hippocampal field. We had originally intended to study this in the striatum, but YFP was not expressed in the striatum of these mice. We therefore focussed on the hippocampal formation as YFP was expressed in this region, and hippocampal deficits have been reported in R6/2 mice. For a recent review, see [36]. We found that YFP intensity is selectively altered in the hippocampal formation of R6/2-YFP-H mice, and that this

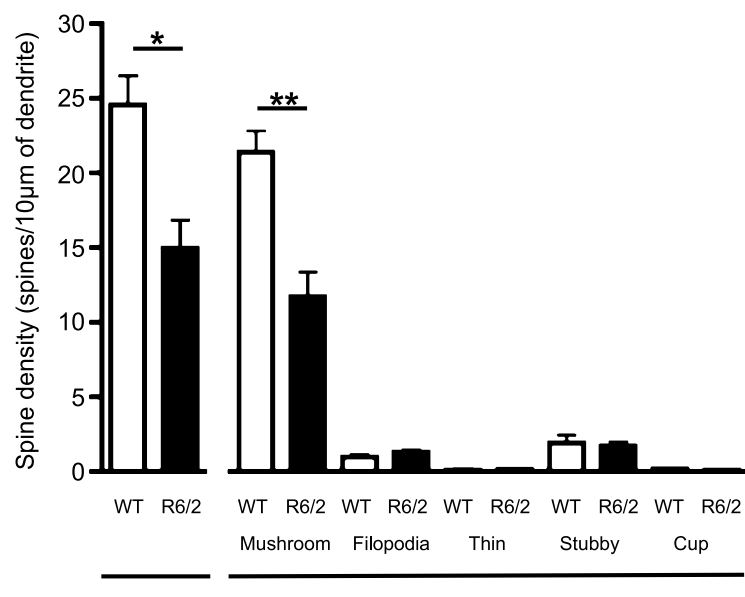

All spines

Spine subclass

Fig. 3. Reduced dendritic spine density in R6/2-YFP-H mice. Mean density $( \pm$ SEM) of different classes of spines from WT-YFP-H and R6/2-YFP-H mice $(* P<0.05 ; * * P<0.01)$. 
is associated with a significant reduction in dendritic spine density in the apical CA1 dendrites, where dysfunctional synaptic plasticity has been observed [7, 25]. Reduced dendritic spine density has previously been reported in striatal neurons from a number of HD mouse models, including the R6/2 model [23, 24, 26, 27] and in human HD [28, 29]. Dendritic spine density has also been found to be reduced in cortical pyramidal neurons of R6/1 mice [21], although a study of prefrontal pyramidal cortical neurons from human HD patients found no difference in dendritic spine density between cases and controls [30]. An important question that remains to be answered is whether changes in spine density contribute to the neurological disturbances that characterise HD. In cultured hippocampal neurons dendritic spines are lost in the proximity of htt aggregates, and preventing this spine loss leads to rescue of neurodegeneration [8].

We did not analyse dendritic spines in the dendate gyrus of R6/2-YFP-H mice, but did observe an increased YFP signal from this region compared to WT-YFP-H mice. Altered synaptic plasticity has been reported in the dentate gyrus of R6/2 mice [7], and it is possible that the increased YFP signal from this region in R6/2-YFP-H mice represents increased spine density. There is evidence that striatal neurons in HD model mice are prone to hyperexcitability and excitotoxicity $[31,32]$, and so it is possible that the changes we observe in CA1 spine density are beneficial compensatory changes in response to hyperexcitability elsewhere in the hippocampal circuit. Consistent with this hypothesis, CA1 neurons from R6/2 mice have previously been found to be resistant to excitotoxic cell death [33]. Setting the changes we have found into the wider context of hippocampal circuitry is an interesting avenue for future work.

A significant increase in the length of mushroomshaped dendritic spines of CA1 pyramidal neurons was found in R6/2-YFP-H mice relative to WT-YFP-H mice. This was unexpected, as reduced dendritic spine length has previously been reported in striatal medium spiny neurons and cortical pyramidal neurons of HD model mice $[23,24]$. In other trinucleotide repeat disorders such as fragile-X mental retardation syndrome, however, an increase in dendritic spine length has been found [24]. It is possible that the spines with increased length seen in R6/2 mice represent immature spines. Synaptic activity is known to be a critical regulator of dendritic spine morphology [34], and during synaptic strengthening spines undergo a transition from appearing long and thin to becoming mushroom-shaped [24]. The increase in spine length we observed may be a correlate of synaptic dysfunction and reduced synaptic plasticity that occurs in human HD and in mouse models of HD [35].

\section{ACKNOWLEDGMENTS}

We thank Wendy Leavens and Zhiguang Zheng for excellent technical support. This work was funded in part by CHDI Inc.

\section{CONFLICT OF INTEREST}

The authors have no conflict of interest to report.

\section{REFERENCES}

[1] Bates G, Harper PS, Jones L. Huntington's Disease, Third edition, Oxford Monographs on Medical Genetics 45. Oxford, UK, Oxford University Press; 2002.

[2] Mangiarini L, Sathasivam K, Seller M, Cozens B, Harper A, Hetherington C, Lawton M, Trottier Y, Lehrach H, Davies SW, Bates GP. Exon 1 of the HD gene with an expanded CAG repeat is sufficient to cause a progressive neurological phenotype in transgenic mice. Cell. 1996;87:493.

[3] Lione LA, Carter RJ, Hunt MJ, Bates GP, Morton AJ, Dunnett SB. Selective discrimination learning impairments in mice expressing the human Huntington's disease mutation. J Neurosci. 1999;19:10428.

[4] Burgess N, Maguire EA, O'Keefe J. The human hippocampus and spatial and episodic memory. Neuron. 2002;35:625.

[5] Koehl M, Abrous DN. A new chapter in the field of memory: Adult hippocampal neurogenesis. Eur J Neurosci. 2011;33:1101.

[6] Malenka RC. The long-term potential of LTP. Nat Rev Neurosci. 2003;4:923.

[7] Murphy KPSJ, Carter RJ, Lione LA, Mangiarini L, Mahal A, Bates GP, Dunnett SB, Morton AJ. Abnormal synaptic plasticity and impaired spatial cognition in mice transgenic for exon 1 of the human Huntington's disease mutation. J Neurosci. 2000;20:5115.

[8] Richards P, Didszun C, Campesan S, Simpson A, Horley B, Young KW, Glynn P, Cain K, Kyriacou CP, Giorgini F, Nicotera P. Dendritic spine loss and neurodegeneration is rescued by Rab11 in models of Huntington's disease. Cell Death Differ. 2011;18:191.

[9] Singaraja RR, Huang K, Sanders SS, Milnerwood AJ, Hines R, Lerch JP, Franciosi S, Drisdel RC, Vaid K, Young FB, Doty C, Wan J, Bissada N, Henkelman RM, Green WN, Davis NG, Raymond LA, Hayden MR. Altered palmitoylation and neuropathological deficits in mice lacking HIP14. Hum Mol Genet. 2011;20:3899.

[10] von Bohlen und Halbach O, Krause S, Medina D, Sciarretta C, Minichiello L, Unsicker K. Regional- and age-dependent reduction in trkB receptor expression in the hippocampus is associated with altered spine morphologies. Biol Psychiatry. 2006;59:793.

[11] Muller D, Toni N, Buchs PA. Spine changes associated with long-term potentiation. Hippocampus. 2000;10:596.

[12] Toni N, Buchs PA, Nikonenko I, Bron CR, Muller D. LTP promotes formation of multiple spine synapses between a single axon terminal and a dendrite. Nature. 1999;402:421. 
[13] Myers RH, Vonsattel J, Stevens TJ, Cupples LA, Richardson EP, Martin JB, Bird ED. Clinical and neuropathologic assessment of severity in Huntington's disease. Neurology. 1988;38:341.

[14] Smith MA, Brandt J, Shadmehr R. Motor disorder in Huntington's disease begins as a dysfunction in error feedback control. Nature. 2000;403:544.

[15] Foroud T, Siemers E, Kleindorfer D, Bill DJ, Hodges ME, Norton J, Conneally PM, Christian JC. Cognitive scores in carriers of Huntington's disease gene compared to noncarriers. Ann Neurol. 1995;37:657.

[16] Lawrence AD, Hodges JR, RosSeries AE, Kershaw A, ffrench-Constant C, Rubinsztein DC, Robbins TW, Sahakian BJ. Evidence for specific cognitive deficits in preclinical Huntington's disease. Brain. 1998;121:1329.

[17] Feng G, Mellor RH, Bernstein M, Keller-Peck C, Nguyen QT, Wallace M, Nerbonne JM, Lichtman JW, Sanes JR. Imaging neuronal subsets in transgenic mice expressing multiple spectral variants of GFP. Neuron. 2000;28:41.

[18] Carter RJ, Lione LA, Humby T, Mangiarini L, Mahal A, Bates GP, Dunnett SB, Morton AJ. Characterisation of progressive motor deficits in mice transgenic for the human Huntington's disease mutation. J Neurosci. 1999;19:3248.

[19] Abramoff MD, Magelhaes PJ, Ram SJ. Image processing with ImageJ. Biophotonics International. 2004;11:36.

[20] Hering H, Sheng M. Dendritic spines: Structure, dynamics and regulation. Nat Rev. Neurosci. 2001;2:880.

[21] Spires TL, Grote HE, Garry S, Cordery PM, Van Dellen A, Blakemore C, Hannan AJ. Dendritic spine pathology and deficits in experience-dependent dendritic plasticity in R6/1 Huntington's disease transgenic mice. Eur J Neurosci. 2004;19:2799.

[22] Sawiak SJ, Wood NI, Williams GB, Morton AJ, Carpenter TA. Voxel-based morphometry in the R6/2 transgenic mouse reveals differences between genotypes not seen with manual 2D morphometry. Neurobiol Dis. 2009;33:20.

[23] Klapstein GJ, Fisher RS, Zanjani H, Cepeda C, Jokel ES, Chesselet MF, Levine MS. Electrophysiological and morphological changes in striatal spiny neurons in R6/2 Huntington's disease transgenic mice. J Neurophysiol. 2001;86:2667.

[24] Irwin SA, Galvez R, Greenough WT. Dendritic spine structural anomalies in fragile-X mental retardation syndrome. Cerebral Cortex. 2000;10:1038.

[25] Lynch G, Kramar EA, Rex CS, Jia Y, Chappas D, Gall $\mathrm{CM}$, Simmons DA. Brain-derived neurotrophic factor restores synaptic plasticity in a knock-in mouse model of Huntington's disease. J Neurosci. 2007;27:4424.
[26] Heck N, Betuing S, Vanhoutte P, Caboche J. A deconvolution method to improve automated 3D-analysis of dendritic spines: Application to a mouse model of Huntington's disease. Brain Struct Funct. 2012;217:421.

[27] Lerner RP, Trejo Martinez Ldel C, Zhu C, Chesselet MF, Hickey MA. Striatal atrophy and dendritic alterations in a knock-in mouse model of Huntington's disease. Brain Res Bull. 2012;87:571.

[28] Graveland GA, Williams RS, DiFiglia M. Evidence for degenerative and regenerative changes in neostriatal spiny neurons in Huntington's disease. Science. 1985;227:770.

[29] Ferrante RJ, Kowall NW, Richradson EP Jr. Proliferative and degenerative changes in striatal spiny neurons in Huntington's disease: A combined study using the section-Golgi method and calbindin D28k immunocytochemistry. J Neurosci. 1991;11:3877.

[30] Sotrel A, Williams RS, Kaufmann WE, Myers RH. Evidence for Neuronal degeneration and dendritic plasticity in cortical pyramidal Neurons of Huntington's disease: A quantitative Golgi study. Neurology. 1993;43:2088

[31] Zeron MM, Hansson O, Chen N, Wellington CL, Leavitt BR, Brundin P, Hayden MR, Raymond LA. Increased sensitivity to N-methyl-D-aspartate receptor-mediated excitotoxicity in a mouse model of Huntington's disease. Neuron. 2002;33:849.

[32] Cepeda C, Hurst RS, Calvert CR, Hernandez-Echeagaray E, Nguyen OK, Jocoy E, Christian LJ, Ariano MA, Levine MS. Transient and progressive electrophysiological alterations in the corticostriatal pathway in a mouse model of Huntington's disease. J Neurosci. 2003;23:961.

[33] Hansson O, Guatteo E, Mercuri NB, Bernardi G, Li XJ, Castilho RF, Brundin P. Resistance to NMDA toxicity correlates with appearance of nuclear inclusions, behavioural deficits and changes in calcium homeostasis in mice transgenic for exon 1 of the huntingtin gene. Eur $\mathrm{J}$ Neurosci. 2001; $14: 1492$.

[34] Harris KM, Fiala JC, Ostroff L. Structural changes at dendritic spine synapses during long-term potentiation. Phil Trans R Soc Lond B. 2003;358:745.

[35] Milnerwood AJ, Raymond LA. Early synaptic pathophysiology in neurodegeneration: Insights from Huntington's disease. Trends Neurosci. 2010;33:513.

[36] Nithianantharajah J, Hannan A, J. Dysregulation of synaptic proteins, dendritic spine abnormalities, and pathological plasticity of synapses as experience-dependent mediators of cognitive and psychiatric symptoms in Huntington's disease. Neuroscience. 2012; in press. 